\title{
Efficiency of Runge-Kutta Methods in Solving Simple Harmonic Oscillators
}

\author{
Annie Gorgey and Nor Azian Aini Mat* \\ Department of Mathematics, Faculty of Science and Mathematics, Universiti Pendidikan Sultan Idris \\ 35900 Tanjong Malim, Perak, Malaysia \\ ${ }^{*}$ Corresponding author: nor.azian@fsmt.upsi.edu.my,
}

Article history

Received: 19 July 2017

Received in revised form: 23 November 2017

Accepted: 27 December 2017

Published on line: 1 June 2018

\begin{abstract}
Symmetric methods such as the implicit midpoint rule (IMR), implicit trapezoidal rule (ITR) and 2-stage Gauss method are beneficial in solving Hamiltonian problems since they are also symplectic. Symplectic methods have advantages over non-symplectic methods in the long term integration of Hamiltonian problems. The study is to show the efficiency of IMR, ITR and the 2-stage Gauss method in solving simple harmonic oscillators (SHO). This study is done theoretically and numerically on the simple harmonic oscillator problem. The theoretical analysis and numerical results on SHO problem showed that the magnitude of the global error for a symmetric or symplectic method with stepsize $h$ is linearly dependent on time $t$. This gives the linear error growth when a symmetric or symplectic method is applied to the simple harmonic oscillator problem. Passive and active extrapolations have been implemented to improve the accuracy of the numerical solutions. Passive extrapolation is observed to show quadratic error growth after a very short period of time. On the other hand, active extrapolation is observed to show linear error growth for a much longer period of time.
\end{abstract}

Keywords Symmetric; symplectic; linear error growth; extrapolation; simple harmonic oscillator.

\section{Mathematics Subject Classification 65L06}

\section{Introduction}

Hamiltonian problems are problems that maintain the structure or behaviour of the numerical solution over long time interval. The Hamiltonian systems are defined as follows:

Let $H: \mathbb{R} \times \mathbb{R}^{n} \times \mathbb{R}^{n} \rightarrow \mathbb{R}$ be a real-valued function. Consider $(t, p, q) \in \mathbb{R} \times \mathbb{R}^{n} \times \mathbb{R}^{n}$, where $p=\left(p_{1}, \cdots, p_{n}\right)$ and $q=\left(q_{1}, \cdots, q_{n}\right)$. Then a $n$-dimensional system is given by

$$
\begin{aligned}
\frac{d q_{i}}{d t} & =\frac{\partial H}{\partial p_{i}} \\
\frac{d p_{i}}{d t} & =-\frac{\partial H}{\partial q_{i}}, \quad i=1, \ldots, n .
\end{aligned}
$$


Both $p$ and $q$ are vector functions of $d$ (number of degrees of freedom of mechanical system). The $q_{i}$ are called generalised coordinates while $p_{i}$ are called the conjugated generalised momenta. $H$ is called the Hamiltonian of the system and for mechanical systems it represents the total mechanical energy [8].

The simplest Hamiltonian system is the linear harmonic oscillator that can be written as the Hamiltonian equation of the motion.

$$
\begin{aligned}
q^{\prime}(t) & =p(t), \\
p^{\prime}(t) & =-q(t),
\end{aligned}
$$

where $y_{0}=\left[q_{0}, p_{0}\right]=[1,0]^{T}$. The exact solution is $y\left(t_{n}\right)=\left[\cos \left(t_{n}\right),-\sin \left(t_{n}\right)\right]$. The exact solution's phase diagram is a unit circle. For the numerical solution to have the same magnitude as the exact solution the magnitude of the stability function on the imaginary axis must satisfy the following condition

$$
|R(i h)|=1
$$

For Runge-Kutta methods to satisfy the condition (2), that methods has to be symmetric and symplectic which are defined in the following section.

\section{Symmetric and Symplectic Runge-Kutta Methods}

\section{$2.1 \quad$ Symmetric}

Let $\mathscr{R}$ denotes the equivalence class of Runge-Kutta methods written as $\mathscr{R}=(A, b, c)$, where $A$ is the Runge-Kutta matrix, $b$ the vector of weights, $c$ the vector of abscissas and $e$ the vector of units. At the $n$th step $x_{n-1} \rightarrow x_{n}=x_{n-1}+h$ with stepsize $h$, the method is defined by

$$
\begin{aligned}
Y^{[n]} & =e \otimes y_{n-1}+h\left(A \otimes I_{N}\right) F\left(x_{n-1}+c h, Y^{[n]}\right), \\
y_{n} & =y_{n-1}+h\left(b^{T} \otimes I_{N}\right) F\left(x_{n-1}+c h, Y^{[n]}\right),
\end{aligned}
$$

for an initial value problem $y^{\prime}(x)=f(x, y), y\left(x_{0}\right)=y_{0} \cdot y_{n}$ is the update while $Y^{[n]}$ is the vector of internal stages with

$$
Y^{[n]}=\left[\begin{array}{c}
Y_{1}^{[n]} \\
\vdots \\
Y_{s}^{[n]}
\end{array}\right], \quad F\left(x_{n-1}+c h, Y^{[n]}\right)=\left[\begin{array}{c}
f\left(x_{n-1}+c_{1} h, Y_{1}^{[n]}\right) \\
\vdots \\
f\left(x_{n-1}+c_{s} h, Y_{s}^{[n]}\right)
\end{array}\right] .
$$

The method is called symmetric or self-adjoint if

$$
-\mathscr{R}^{-1}=\mathscr{R}
$$

where $-\mathscr{R}=(-A,-b,-c)$ is the method $\mathscr{R}$ applied with stepsize $-h$, and $\mathscr{R}^{-1}=(A-$ $\left.e b^{T},-b, c-e\right)$ is the inverse of $\mathscr{R}$. It follows that the adjoint of $\mathscr{R}$ is given by $-\mathscr{R}^{-1}=$ $\left(e b^{T}-A, b, e-c\right) \equiv(P A P, P b, P c)$, and a symmetric method is therefore characterized by the symmetry conditions,

$$
P b=b, \quad P A P=e b^{T}-A, \quad P c=e-c,
$$


where $P$ is a permutation matrix which reverses the order of the stages with its elements satisfying $p_{i j}=\delta_{i, s+1-j}$. The third condition assumes that $b^{T} e=1$ and $A e=c$ hold. To show that symmetric methods satisfy condition (2), it can be shown that the stability function $R(z)=1+z b^{T}(I-z A)^{-1} e$ satisfies

$$
R(z) R(-z)=1
$$

When $z=i h, R(-i h)=\overline{R(i h)}$ so that $|R(i h)|=1$.

There are also symmetrized methods studied by Chan and Gorgey [4] and [7] that are not symmetric itself but do posses the asymptotic error expansions of even powers.

\subsection{Symplectic}

For an s-stage Runge-Kutta method (3) to be symplectic [12], the condition needed to be satisfied is

$$
B A+A^{T} B=b b^{T}
$$

where $B$ is a matrix with elements of $b$ on the diagonal position, that is defined as follows:

$$
B(i, j)= \begin{cases}b_{i}, & \text { if } i=j \\ 0, & \text { otherwise }\end{cases}
$$

If the RK method is symplectic, then its stability function also satisfies condition (2).

For example the ITR, IMR and 2-stage Gauss method are symplectic and symmetric by (2) and (7) and they are defined in Table 1.

Table 1: Some Examples of Symmetric and Symplectic Runge-Kutta Methods

$$
\begin{array}{c|cc|ccc|cc}
\frac{1}{2} & \frac{1}{2} & & 0 & 0 & \frac{1}{2}-\frac{\sqrt{3}}{6} & \frac{1}{4} & \frac{1}{4}-\frac{\sqrt{3}}{6} \\
\hline & 1 & & \frac{1}{2} & \frac{1}{2} & \frac{1}{2}+\frac{\sqrt{3}}{6} & \frac{1}{4}+\frac{\sqrt{3}}{6} & \frac{1}{4} \\
\hline & \frac{1}{2} & \frac{1}{2} & & \frac{1}{2} & \frac{1}{2}
\end{array}
$$
(a) IMR
(b) ITR
(c) 2-stage Gauss

To show that the Gauss 2-stage method is symplectic, consider the following example.

Consider the 2-stage Gauss method defined in Table 1.

$$
A=\left(\begin{array}{cc}
\frac{1}{4} & \frac{1}{4}-\frac{\sqrt{3}}{6} \\
\frac{1}{4}+\frac{\sqrt{3}}{6} & \frac{1}{4}
\end{array}\right), B=\left(\begin{array}{cc}
\frac{1}{2} & 0 \\
0 & \frac{1}{2}
\end{array}\right) .
$$

To check whether the method is symplectic, solve for $B A+A^{T} B=b b^{T}$.

LHS gives,

$$
B A+A^{T} B=\left(\begin{array}{cc}
\frac{1}{8} & \frac{1}{8}-\frac{\sqrt{3}}{12} \\
\frac{1}{8}+\frac{\sqrt{3}}{12} & \frac{1}{8}
\end{array}\right)+\left(\begin{array}{cc}
\frac{1}{8} & \frac{1}{8}+\frac{\sqrt{3}}{12} \\
\frac{1}{8}-\frac{\sqrt{3}}{12} & \frac{1}{8}
\end{array}\right)=\left(\begin{array}{cc}
\frac{1}{4} & \frac{1}{4} \\
\frac{1}{4} & \frac{1}{4}
\end{array}\right)
$$


Similarly, solving for RHS gives

$$
b b^{T}=\left(\begin{array}{c}
\frac{1}{2} \\
\frac{1}{2}
\end{array}\right)\left(\begin{array}{ll}
\frac{1}{2} & \frac{1}{2}
\end{array}\right)=\left(\begin{array}{cc}
\frac{1}{4} & \frac{1}{4} \\
\frac{1}{4} & \frac{1}{4}
\end{array}\right),
$$

Hence, Gauss 2-stage method satisfies the symplectic condition.

Symplectic numerical methods exist for reliable long time integration of Hamiltonian systems. Sanz- Serna [12] has systematically developed symplectic Runge- Kutta (RK) methods. Their idea is based on features of algebraic stability introduced, in connection with stiff systems studied by Burrage and Butcher [2]. Such methods have wide range of applications not only in Hamiltonian problems but also in optimal control problems and in other applications requiring the use of adjoint systems [11]. A group of Chinese mathematicians [16] have studied the stochastic symplectic Runge-Kutta methods for strong approximations of Hamiltonian systems with additive noise both theoretically and numerically. From their observation, when solving stochastic harmonic oscillator with additive noise, the linear growth property can be preserved exactly over long-time simulation.

\section{Error Analysis on Simple Harmonic Oscillator (SHO)}

The SHO problem can be expressed as the initial value problem (IVP) by using complex numbers and denoting $y=p+i q$ which is given as follows:

$$
y^{\prime}=i y, \quad y(0)=y_{0},
$$

where $y_{0}$ is the starting value and the exact solution is given by

$$
y\left(t_{n}\right)=y_{0} e^{i t_{n}}
$$

Applying RK method (3) that has the stability function $R(z)=I+z b^{T}(I-z A)^{-1} e$ using a constant stepsize $h$ to (8) gives the numerical solution at time $t_{n}=n h$,

$$
y_{h}\left(t_{n}\right)=R(i h) y_{h}\left(t_{n-1}\right)=R(i h)^{n} y_{0}
$$

with $y_{h}(0)=y_{0}$.

By the principle of logarithm since $R(i h)=e^{\log R(i h)}=e^{\log |R(i h)|+i \arg R(i h)}$. Defining

$$
\phi(h)=\frac{1}{h}(\arg R(i h)-i \log |R(i h)|),
$$

gives

$$
R(i h)=e^{i h \phi(h)},
$$

so that

$$
y_{h}\left(t_{n}\right)=e^{i t_{n} \phi(h)} y_{0} .
$$


The initial value is given by $y_{0}=e^{-i t_{n}} y\left(t_{n}\right)$. Hence the global error at time $t_{n}$ is given by

$$
y_{h}\left(t_{n}\right)-y\left(t_{n}\right)=\left(e^{i \theta(h)}-1\right) y\left(t_{n}\right)
$$

where $\theta(h)=t_{n}(\phi(h)-1)$.

Basically the performance of the numerical solutions depends on how well the $\phi(h)$ function behaves. If $\phi(h)$ is real then only the phase is affected. If $\phi(h)$ is purely imaginary then the amplitude is affected. In general both the phase and amplitude of the exact solution are modified by the numerical solution.

Lemma 1 If the method is symmetric and symplectic, then $\phi(h)$ is a real and even function such that for $\phi(h)$ to be an even function, $|R(i h)|=1$ ([3]).

To define the $\phi(h)$ function given in (10) for Runge-Kutta methods, consider modifying the stability function $R(z)$ for $R(i h)$.

$$
\begin{aligned}
R(z) & =1+z b^{T}(I-z A)^{-1} e, \\
R(i h) & =1+(i h) b^{T}(I-i h A)^{-1} e, \\
& =1+(i h) b^{T}(I-i h A)^{-1}(I+i h A)^{-1}(I+i h A)^{-1} e, \\
& =1-h^{2} b^{T}\left(I+h^{2} A^{2}\right)^{-1} A e+i h b^{T}\left(I+h^{2} A^{2}\right)^{-1} e .
\end{aligned}
$$

Taking the complex arguments yields

$$
\arg (R(i h))=\tan ^{-1}\left(\frac{h b^{T}\left(I+h^{2} A^{2}\right)^{-1} e}{1-h^{2} b^{T}\left(I+h^{2} A^{2}\right)^{-1} A e}\right) .
$$

From (10), and by Lemma 1

$$
\phi(h)=\frac{1}{h} \tan ^{-1}\left(\frac{h b^{T}\left(I+h^{2} A^{2}\right)^{-1} e}{1-h^{2} b^{T}\left(I+h^{2} A^{2}\right)^{-1} A e}\right) .
$$

Example 1 For IMR with $A=1 / 2, b=1$ and $e=1$,

$$
\begin{aligned}
\phi(h) & =\frac{1}{h} \tan ^{-1}\left(\frac{h\left(I+\frac{h^{2}}{4}\right)^{-1}}{1-h^{2}\left(I+\frac{h^{2}}{4}\right)^{-1} \frac{1}{2}}\right), \\
& =\frac{1}{h} \tan ^{-1}\left(\frac{h}{1-\frac{h^{2}}{4}}\right)=1-\frac{1}{12} h^{2}+\frac{1}{80} h^{4}-\frac{1}{448} h^{6}+O\left(h^{8}\right) .
\end{aligned}
$$

A similar $\phi(h)$ function is obtained for symmetrized IMR that is given in [4]. Although the IMR is symmetric and symplectic, but the symmetrized IMR is neither symmetric nor symplectic. However it is interesting to know that they have similar $\phi(h)$ function.

Example 2 For ITR, the $\phi(h)$ function is given as

$$
\phi(h)=\frac{1}{h} \tan ^{-1}\left(\frac{h}{1-\frac{h^{2}}{2}}\right)=1+\frac{1}{6} h^{2}-\frac{1}{20} h^{4}-\frac{1}{56} h^{6}+O\left(h^{8}\right) .
$$


Example 3 For 2-stage Gauss method as given in Table 1, using Maple 2016, the equation is expanded in series and the $\phi(h)$ function is given as

$$
\phi(h)=\frac{1}{h} \tan ^{-1}\left(\frac{12\left(h^{2}-12\right) h}{h^{4}-60 h^{2}+144}\right)=1-\frac{1}{720} h^{4}+\frac{1}{12096} h^{6}+O\left(h^{8}\right) .
$$

In order to understand the behaviour of symplectic and non-symplectic Runge-Kutta methods, a simple experiment has been carried out and the numerical results are given in section Numerical Results.

There are also combination of two methods that can be shown to be symmetric and symplectic. The combinations of these two methods is known as partitioned Runge-Kutta methods (PKR), as given in [1] and [14]. PKR methods is advantageous in solving Hamiltonian system that is separable such as given in (3). A PKR method consists of two RK methods where one method solve for $p$ and the other method solve for $q$. One example of PKR method is the Stömer-Verlet (SV) method which consists of 2-stage Lobatto IIIA and Lobatto IIIB methods.

The construction of symplectic (partitioned) Runge-Kutta methods with continuous stage are studied by Tang, Lang and Luo [15], recently. By relying on the extension of the orthogonal polynomials and simplifying assumptions these partitioned RK methods are constructed. In addition to that, the construction of trigonometrically fitted symplectic Runge-Kutta-Nystrm (RKN) methods from symplectic trigonometrically fitted partitioned. Runge-Kutta (PRK) methods up to five stages and fourth algebraic order are investigated by Monovasilis, Kalogiratou and Simos [10] for the two-body problem and the perturbed two-body problem.

\section{Linear Error Growth for Symmetric and Symplectic Methods}

The numerical solution of the base method with stepsize $h$ at time $t_{n}$ is given by

$$
y_{h}\left(t_{n}\right)=e^{i \theta(h)} y\left(t_{n}\right) .
$$

For a symmetric or symplectic method, $\phi(h)=\phi(i h)=\frac{1}{h} \arg R(i h)$. It is observed that (refering to Example 1-Example 3), when $\phi$ is even and real function, it has the expansion in the following form:

$$
\phi(i h)=1+c_{p}(i h)^{p}+c_{p+2}(i h)^{p+2}+O\left((i h)^{p+4}\right)
$$

where $c_{p}, c_{p+2}, \ldots$, are constants independent of $h$.

Since $\theta(h)=t_{n}(\phi(h)-1)$, substituting (14) gives

$$
\begin{aligned}
\theta(h) & =t_{n}(\phi(h)-1), \\
& =t_{n}\left(c_{p}(i h)^{p}+c_{p+2}(i h)^{p+2}+O\left((i h)^{p+4}\right)\right), \\
& =i^{p}\left(c_{p} \tau-c_{p+2} \tau h^{2}+O\left(\tau h^{4}\right)\right), \text { where } t_{n} h^{p}=\tau .
\end{aligned}
$$


The global error is given by

$$
\begin{aligned}
\epsilon_{h}\left(t_{n}\right) & =y_{h}\left(t_{n}\right)-y\left(t_{n}\right), \\
& =\left(e^{i \theta(h)}-1\right) y\left(t_{n}\right), \\
& =e^{i \frac{\theta(h)}{2}}\left(e^{i \frac{\theta(h)}{2}}-e^{-i \frac{\theta(h)}{2}}\right) y\left(t_{n}\right), \\
& =e^{i \frac{\theta(h)}{2}}\left(2 i \sin \left(\frac{\theta(h)}{2}\right)\right) y\left(t_{n}\right) .
\end{aligned}
$$

The magnitude of the global error for a symmetric or symplectic method is given by

$$
\left|\epsilon_{h}\left(t_{n}\right)\right|=2\left|\sin \left(\frac{\theta(h)}{2}\right)\right| y\left(t_{n}\right) .
$$

Assuming $\left|y\left(t_{n}\right)\right|=\left|y_{0}\right|=1$ and $\theta(h)$ is small, the Taylor series expansion of $\sin \left(\frac{\theta(h)}{2}\right)$ gives

$$
\begin{aligned}
2\left|\sin \left(\frac{\theta(h)}{2}\right)\right| & =2\left|\frac{\theta(h)}{2}-\frac{1}{6}\left(\frac{\theta(h)}{2}\right)^{3}+O(\theta(h))^{5}\right|, \\
& =\left|\theta(h)-\frac{1}{24}(\theta(h))^{3}+O(\theta(h))^{5}\right| .
\end{aligned}
$$

Hence the global error is obtained by substituting (15) into (18).

$$
\begin{aligned}
\left|\epsilon_{h}\left(t_{n}\right)\right| & =\left|\theta(h)-\frac{1}{24}(\theta(h))^{3}+O(\theta(h))^{5}\right| y\left(t_{n}\right), \\
& \leq\left|i^{p} c_{p}\right| \tau-\left|i^{p} c_{p+2}\right| \tau h^{2}+O\left(\tau^{3}+\tau h^{4}\right), \\
& =\left|c_{p}\right| \tau+O\left(\tau^{2}+\tau h^{3}\right) .
\end{aligned}
$$

Note: The term $O\left(\tau^{3}\right)$ is obtained by expanding $(\theta(h))^{3}$ in series and considering only the first term.

From (19), if $\tau$ and $h$ are relatively small then the global error is dominated by $\left|c_{p}\right| \tau$. Since $\tau=t_{n} h^{p}$, the magnitude of the global error for a symmetric or symplectic method with stepsize $h$ is linearly dependent on time $t$. This gives the linear error growth when a symmetric or symplectic method is applied to the simple harmonic oscillator problem.

\section{$5 \quad$ Numerical Results}

Figure 1-3 give the numerical results of IMR, ITR and 2-stage Gauss methods with passive and active extrapolation applied to simple harmonic oscillator at different time. At $t_{n}=10$, it is shown that all these methods exhibits linear error growth as shown theoretically. The numerical results were also given for passive and active extrapolation. The theoretical results for symmetric or symplectic methods when applied with passive and active extrapolation is given in another paper by the author [6]. The numerical results for active extrapolation is observed to give the lowest error if compared with passive extrapolation. As the time increases $\left(t_{n}=1000\right)$, it is shown in Figure 2 that passive extrapolation gives quadratic error growth. However, active extrapolation still gives the lowest error even with linear error growth. Figure 3 shows the numerical results at $t_{n}=1000000$. All the base methods with passive extrapolation give periodic behaviour except for active extrapolation for IMR and ITR. 

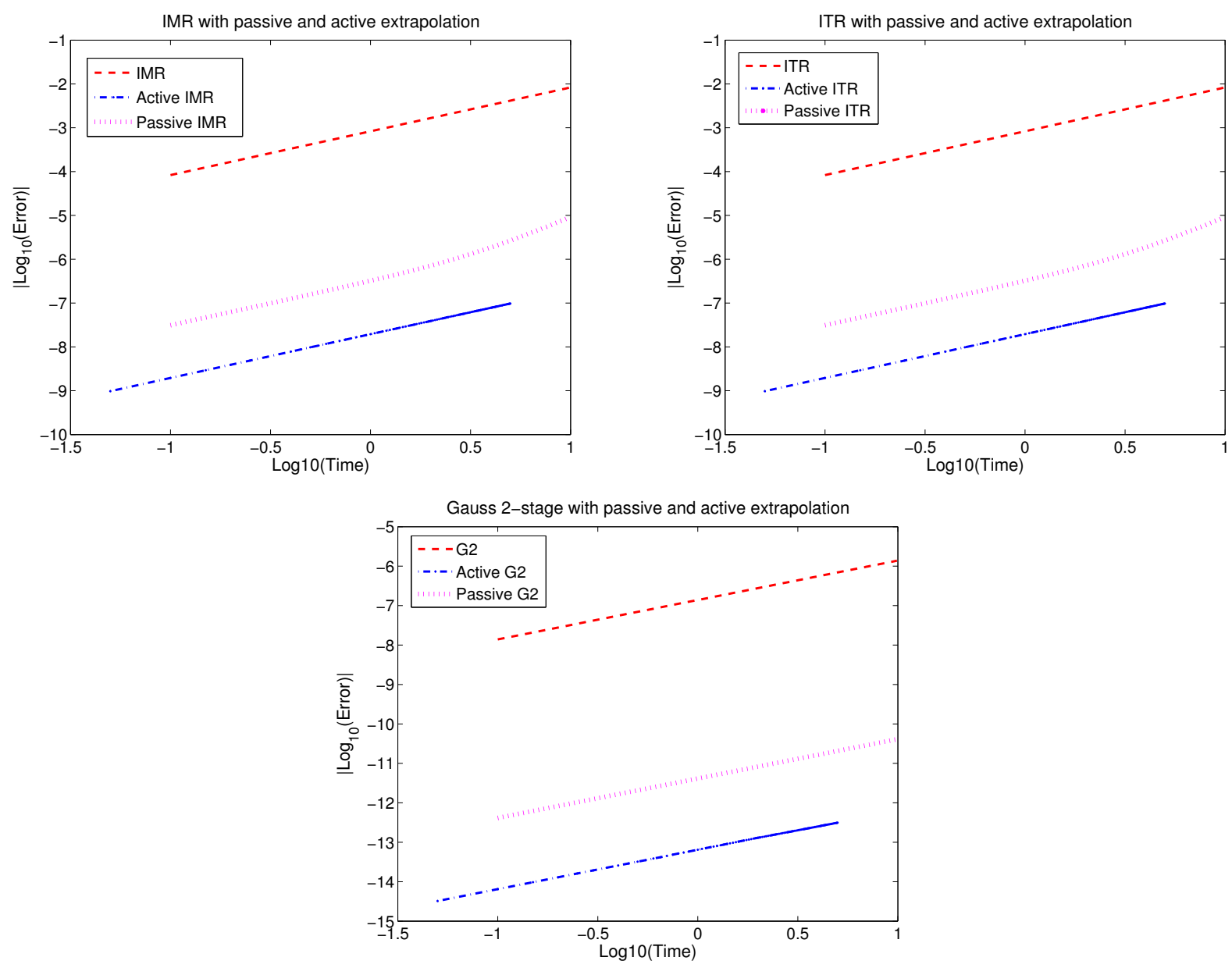

Figure 1: The Linear Error Growth of IMR, ITR and 2-stage Gauss Methods with Passive and Active Extrapolation in Solving Simple Harmonic Oscillator for $h=0.1$ at $t_{n}=10$. 

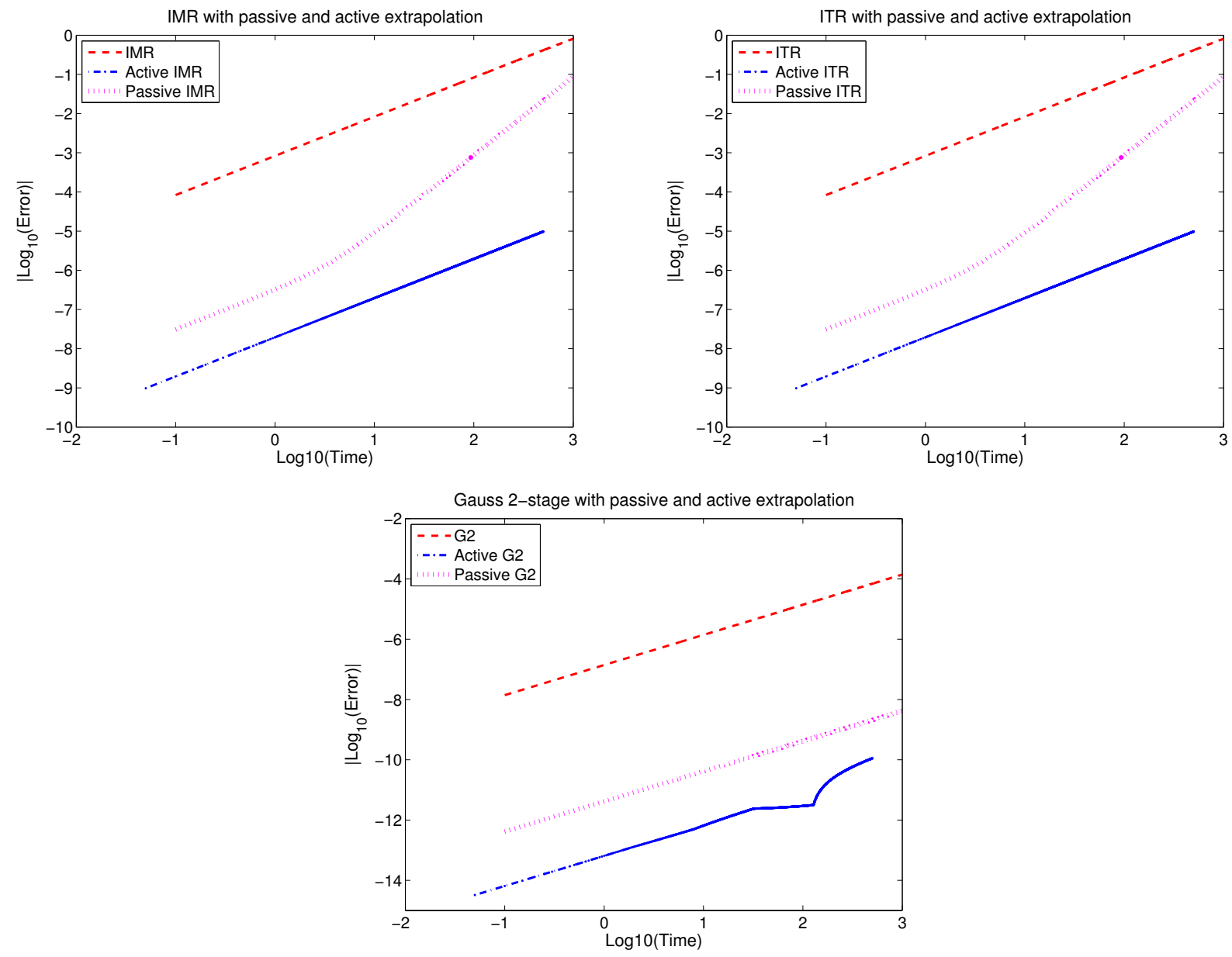

Figure 2: The linear error growth of IMR, ITR and 2-stage Gauss methods with passive and active extrapolation in solving Simple Harmonic Oscillator for $h=0.1$ at $t_{n}=1000$. 

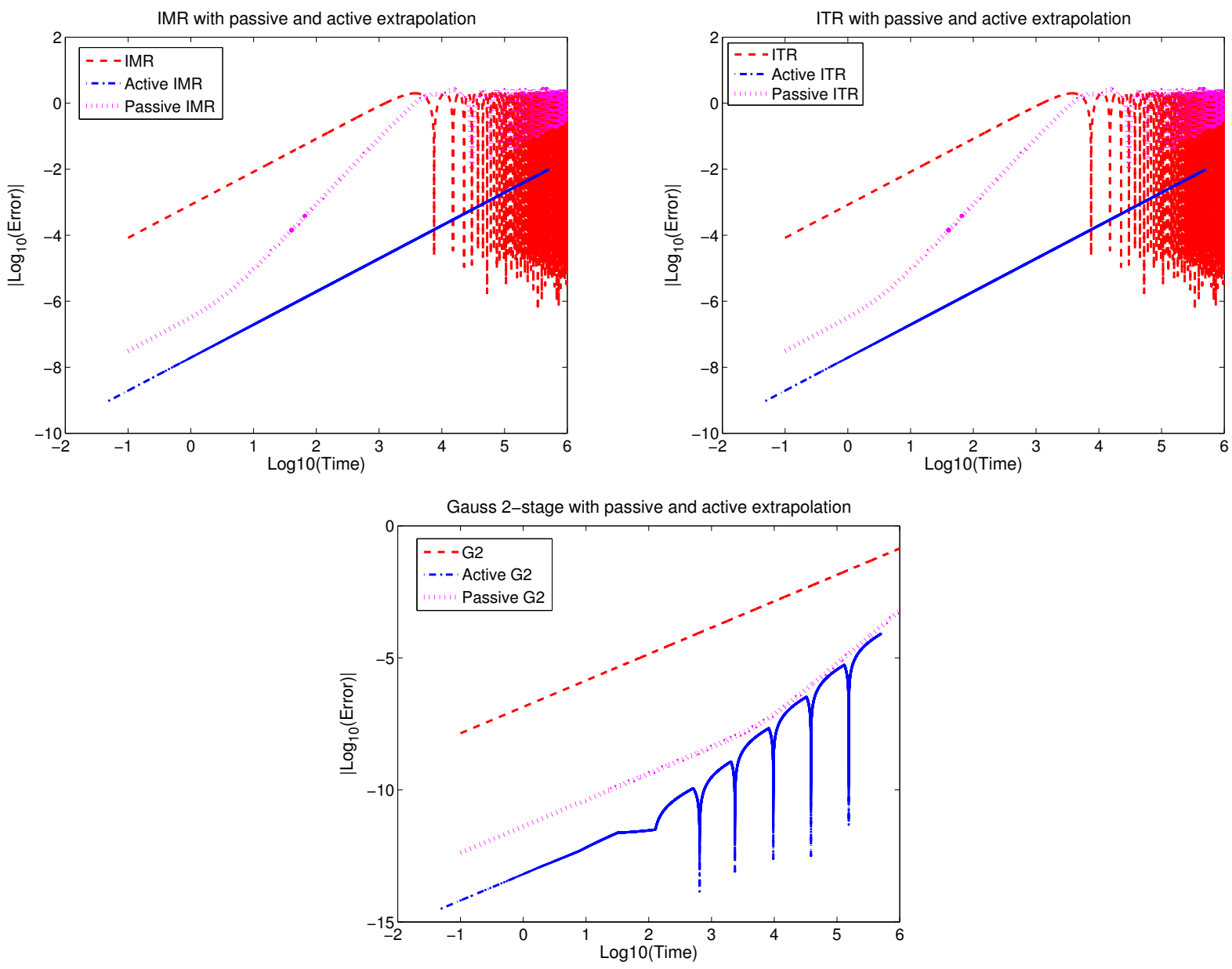

Figure 3: The linear error growth of IMR, ITR and 2-stage Gauss methods with passive and active extrapolation in solving Simple Harmonic Oscillator for $h=0.1$ at $t_{n}=1000000$. 


\section{Conclusions}

Symmetric methods such as the IMR, ITR and 2-stage Gauss are beneficial in solving Hamiltonian problems since they are also symplectic. Symplectic methods have advantages over non-symplectic methods in the long term integration of Hamiltonian problems. For instant, in the simple harmonic oscillator problem, symplectic methods such as the IMR, ITR and 2-stage Gauss show linear error growth. Passive and active extrapolations have been implemented to improve the accuracy of the numerical solutions. Passive extrapolation is observed to show quadratic error growth after a very short period of time. On the other hand, active extrapolation is observed to show linear error growth for a much longer period of time. In addition to that, active extrapolation is also give stable results for a longer period of time and therefore is more advantageous on solving simple harmonic oscillator especially for IMR and ITR. However, for 2-stage Gauss method it is observed that active extrapolation gives oscillatory behaviour over long time interval. We wish to extend this study with symmetrized methods [5] and hope symmetrization of active and passive extrapolation gives satisfying results.

\section{Acknowledgments}

The authors would like to thank the Universiti Pendidikan Sultan Idris for providing the research grant GPU, Vote No: 2016-0186-102-01.

\section{References}

[1] Abia, L. and Sanz-Serna, J. M. Partitioned Runge-Kutta methods for separable Hamiltonian problems. Mathematics Computations. 1993. 60: 617-634.

[2] Burrage, K. and Butcher, J. C. Stability criteria for implicit Runge-Kutta methods. SIAM Journal of Numerical Analysis. 1979. 16: 46-57.

[3] Chan, R. P. K. and Murua, A. Extrapolation of symplectic methods for Hamiltonian problems. Applied Numerical Mathematics. 2000. 34: 189-205.

[4] Chan, R. P. K. and Gorgey, A. Active and passive symmetrization of Runge-Kutta Gauss methods. Applied Numerical Mathematics. 2013. 67: 64-77.

[5] Gorgey, A. and Chan, R. P. K. Choice of strategies for extrapolation with symmetrization in the constant stepsize setting. Applied Numerical Mathematics. 2015. 87: 31-37.

[6] Gorgey, A. Extrapolation of Runge-Kutta methods in solving simple harmonic oscillators and simple pendulum problems. International Journal of Mathematics and Computation. 2018. $29(2)$.

[7] Gorgey, A. Extrapolation of symmetrized Runge-Kutta methods in the variable stepsize setting, International Journal of Applied Mathematics and Statistics, 2016. 55(2): 1-9.

[8] Hairer, E., Wanner, G. and Lubich, C. Geometric Numerical Integration: StructurePreserving Algorithms for Ordinary Differential Equations, Springer Series in Computational Mathematics. Second Edition. 4. Berlin Heidelberg: Springer-Verlag. 2006. 
[9] Iserles, A. A First Course in the Numerical Analysis of Differential Equations. Second Ed., Cambridge Text in Applied Mathematics. Cambridge University Press. 2008.

[10] Monovasilis, T., Kalogiratou, Z. and Simos, T. E. Construction of exponentially fitted symplectic Runge-Kutta Nystrm methods from partitioned Runge-Kutta Methods. Mediterranean Journal of Mathematics. 2016. 13(4): 2271-2285.

[11] Sanz-Serna, J. M. Symplectic Runge-Kutta schemes for adjoint equations, automatic differentiation, optimal control, and more. SIAM Review, 2016. 58(1): 3-33.

[12] Sanz-Serna, J. M. Runge-Kutta Schemes for Hamiltonian systems. BIT. 1988. 28: 877-883.

[13] Sanz-Serna, J. M. and Calvo, M. P. Numerical Hamiltonian problems. Applied Mathematics Chapman and Mathematical Computational. 1994. 7: 500-510.

[14] Sun, G. A . Simple way constructing symplectic Runge-Kutta methods. Journal of Computational Mathematics. 2000. 18(1): 61-68.

[15] Tang, W., Lang, G. and Luo, X. Construction of symplectic (partitioned) Runge-Kutta methods with continuous stage. Applied Mathematics and Computations. 2016. 286: 279287.

[16] Zhou, W., Zhang, J., Hong, J., and Song, S. Stochastic symplectic Runge-Kutta methods for the strong approximation of Hamiltonian systems with additive noise. Numerical Analysis. 2016.1: 1-24. 